Margaritis Avgeris, Konstantinos Stravodimos and Andreas Scorilas*

\title{
Loss of miR-378 in prostate cancer, a common regulator of $K L K 2$ and $K L K 4$, correlates with aggressive disease phenotype and predicts the short-term relapse of the patients
}

\begin{abstract}
A large number of prostate cancer (PCa) patients receive treatment without significant benefits, strengthening the need for accurate prognosis, which can be supported by the study of miRNAs. In silico specificity analysis was performed for the identification of miRNAs able to regulate $K L K 2$ and $K L K 4$ expression. Total RNA was extracted from prostate tissues obtained from $\mathrm{PCa}$ and benign prostate hyperplasia patients. Thereafter, RNA was polyadenylated and reverse transcribed to cDNA, which was used for qPCR analysis. miR-378 was predicted to target both KLK2 and KLK4 and downregulated levels detected in PCa patients $(p=0.050)$. The reduction of miR-378 was correlated with higher Gleason score $(p=0.018)$, larger diameter tumors $(p=0.034)$, and elevated serum PSA $(p=0.006)$. Regarding prognosis, miR-378 was able to improve risk stratification according to Gleason score or tumor stage, while higher risk to recur highlighted for the patients expressing lower miR-378 levels. Finally, the loss of miR-378 was able to predict the short-term relapse of 'high'- and 'very high'recurrence-risk patients, independent of Gleason score, tumor stage, PSA, and age as indicated by Kaplan-Meier survival curves $(p=0.030)$ and multivariate Cox regression analysis $(p=0.018)$. In conclusion, loss of miR-378 expression increases the risk for PCa progression and relapse, despite active treatment.
\end{abstract}

Keywords: kallikrein-related peptidase; KLK; miR-378a; miR-422a; molecular tumor markers; prostate tumors.

\footnotetext{
*Corresponding author: Andreas Scorilas, Department of Biochemistry and Molecular Biology, University of Athens, Panepistimiopolis, 15701 Athens, Greece,

e-mail: ascorilas@biol.uoa.gr
}

Margaritis Avgeris: Department of Biochemistry and Molecular Biology, University of Athens, Panepistimiopolis, 15701 Athens, Greece

Konstantinos Stravodimos: First Department of Urology, "Laiko" General Hospital, Medical School, University of Athens, Agiou Thoma 17, 11527 Athens, Greece
DOI 10.1515/hsz-2014-0150

Received February 19, 2014; accepted July 3, 2014

\section{Introduction}

Despite the ongoing debate about the reduction of prostate cancer $(\mathrm{PCa})$ mortality, the introduction of prostatespecific antigen (PSA) screening of the male population is primarily responsible for the high rate of $\mathrm{PCa}$ diagnosis in Western populations. However, this beneficial use of PSA, as kallikrein-related peptidase 3 (KLK3) is largely known, suffers from a high rate of overdiagnosis and overtreatment (Andriole et al., 2009; Schroder et al., 2009).

$\mathrm{PCa}$ is characterized by great heterogeneity in terms of disease progression rate and life-threatening potential. Prostate tumors range from clinically insignificant and not life threatening to rapidly progressive and lethal ones, despite active treatment. Consequently, a large number of patients receive radical therapy without important clinical benefits, which further worsens health-related quality of life. Risk stratification and treatment decisions are mainly determined by Gleason score, pathological stage, serum PSA levels, and patient's life expectancy (Freedland, 2011). However, the diverse outcomes of the same risk group of patients clearly highlight the need for a more accurate risk stratification and prognosis of the diagnosed patients, as well as more personalized patient's management.

KLK2 and KLK4 are two highly expressed members of the kallikrein-related peptidases $(K L K)$ gene family in the prostate gland, encoding for KLK2- and KLK4-secreted serine proteases, both responsible for semen liquefaction (Borgono et al., 2004). KLK2 and KLK4 are significantly overexpressed in PCa compared to benign or normal epithelium (Darson et al., 1997; Herrala et al., 2001; Klokk et al., 2007; Avgeris et al., 2011), facilitating prostate tumorigenesis and cancer cells spread. KLK2 and KLK4 were able to deliver tumorigenic stimuli in prostate cells by the overactivation of the insulin-like growth factor (IGF) axis, 
through IGF-binding protein (IGFBP) degradation, the proteolytic cleavage of cell surface proteinase-activated receptors 1 and 2 (PAR1 and 2) and the stimulation of AR and mTOR signaling in PCa cells. Moreover, the KLK2and KLK4-mediated cleavage of extracellular matrix (ECM) components facilitates cancer cell invasiveness and bone metastasis (Avgeris et al., 2010, 2012; Mavridis and Scorilas, 2010). Therefore, the regulation of KLK2 and KLK4 expression is critical for prostate homeostasis and the progression of prostate malignancy.

Although the direct AR-mediated regulation of $K L K$ transcription in prostate gland is well documented, and several androgen response elements (AREs) have been identified in the promoter regions of KLK2 and KLK4 (Lawrence et al., 2010), recent findings have drawn attention to miRNAs as a novel posttranscriptional regulators of KLK expression (Chow et al., 2008; Pasic et al., 2012). Taking into account the deregulated levels of several miRNAs in PCa (Porkka et al., 2007; Schaefer et al., 2010a; Wach et al., 2012), the identification of miRNAs targeting KLK2 and KLK4 and the evaluation of their clinical significance for the patients would further support the need for improvement of disease prognosis.

In the present study, in an effort to improve the risk stratification of the PCa patients and the prediction accuracy of their survival outcome following treatment, we have identified, in silico, hsa-miRNA-378-3p (miR-378) as a common regulator of $K L K 2$ and $K L K 4$ expression, and thereafter, we analyzed its expression levels in PCa.

\section{Results}

\section{miR-378-3p predicted to target KLK2 and KLK4 expression}

Several miRNAs were predicted to bind on the $3^{\prime}$-UTR of $K L K 2$ and KLK4 mRNAs and, thus, to regulate their levels. In order to achieve a good balance between sensitivity and specificity, we focused on those miRNAs predicted to target KLK2 and KLK4 by multiple programs. The miRNAs predicted to regulate both KLK2 and KLK4 by three or more programs are presented in Table 1 . As shown in Table 1, hsa-miR-378-3p and hsa-miR-422a, a paralog of hsa-miR-378-3p, are the most probable candidates to target both $K L K 2$ and $K L K 4$. The several variants of hsa-miR-378-3p (miR-378a-j and miR-422a), although they are encoded by different stem-loop precursors, share the same seed sequence and, therefore, mRNA targets. Among them, hsa-miR-378a-3p, previously annotated as
hsa-miR-422b, is the predominantly expressed variant of the miR-378/422a family. Taking into consideration the increased transcriptional levels of KLK2 and KLK4 and the reduced miR-378 levels in prostate malignancy (MartensUzunova et al., 2012), we decided to investigate miR-378 clinical significance for PCa patients.

\section{Expression analysis of miR-378 in prostate tumors}

Decreased miR-378 levels ( $p=0.050$; Supplemental Figure 1) were detected in PCa (range: 0.10-62.63 RQ units; median: $2.80 \mathrm{RQ}$ units) compared to BPH patients (range: 0.32-113.97 RQ units; median: 3.87 RQ units). ROC analysis highlighted the clinical value of miR-378 in prostate malignancy to discriminate the PCa patients from the BPH ones (AUC: 0.597; 95\% CI: 0.503-0.692; $p=0.050$; Supplemental Figure 1), which was further confirmed by univariate logistic regression analysis (OR: 0.487; 95\% CI: 0.243-0.977; $p=0.043)$.

\section{Downregulation of miR-378 correlates with tumors of higher Gleason score and larger diameter as well as elevated serum PSA levels}

In order to evaluate the prognostic significance of miR-378 for PCa, the miR-378 expression levels were analyzed relatively to the classical and clinically used prognostic markers. A statistically significant $(p=0.018)$ downregulation of miR-378 was observed as the disease progressed to poorly differentiated tumors (Figure 1A). More precisely, significantly lower miR-378 levels were found in Gleason score $\geq 8$ (median: 1.20 RQ units) and Gleason score 7 (median: $2.11 \mathrm{RQ}$ units) tumors compared to those of Gleason score $\leq 6$ (median: $4.37 \mathrm{RQ}$ units). Additionally, the reduction of miR-378 in PCa was found to correlate with higher PSA serum levels as significantly lower ( $p=0.006)$ miR-378 expression was detected in PSA $\geq 10 \mu \mathrm{g} / 1$ cohort (median: $1.34 \mathrm{RQ}$ units) compared to 4-10 $\mu \mathrm{g} / \mathrm{l}$ (median: $4.08 \mathrm{RQ}$ units) and $<4 \mu \mathrm{g} / \mathrm{l}$ (median: 11.06 RQ units) cohorts (Figure 1B). This negative correlation between miR-378 and PSA levels was also supported by Spearman's correlation coefficient $\left(\mathrm{r}_{\mathrm{s}}:-0.273 ; p=0.021\right.$; Figure 1C). Finally, miR-378 expression was also found to correlate with larger tumor diameter in PCa patients, as illustrated by Spearman's correlation $\left(\mathrm{r}_{\mathrm{s}}:-0.402 ; p=0.034\right.$; Figure 1D). However, no statistically significant correlation was observed between miR-378 levels and tumor stage (Supplemental Figure 2). 


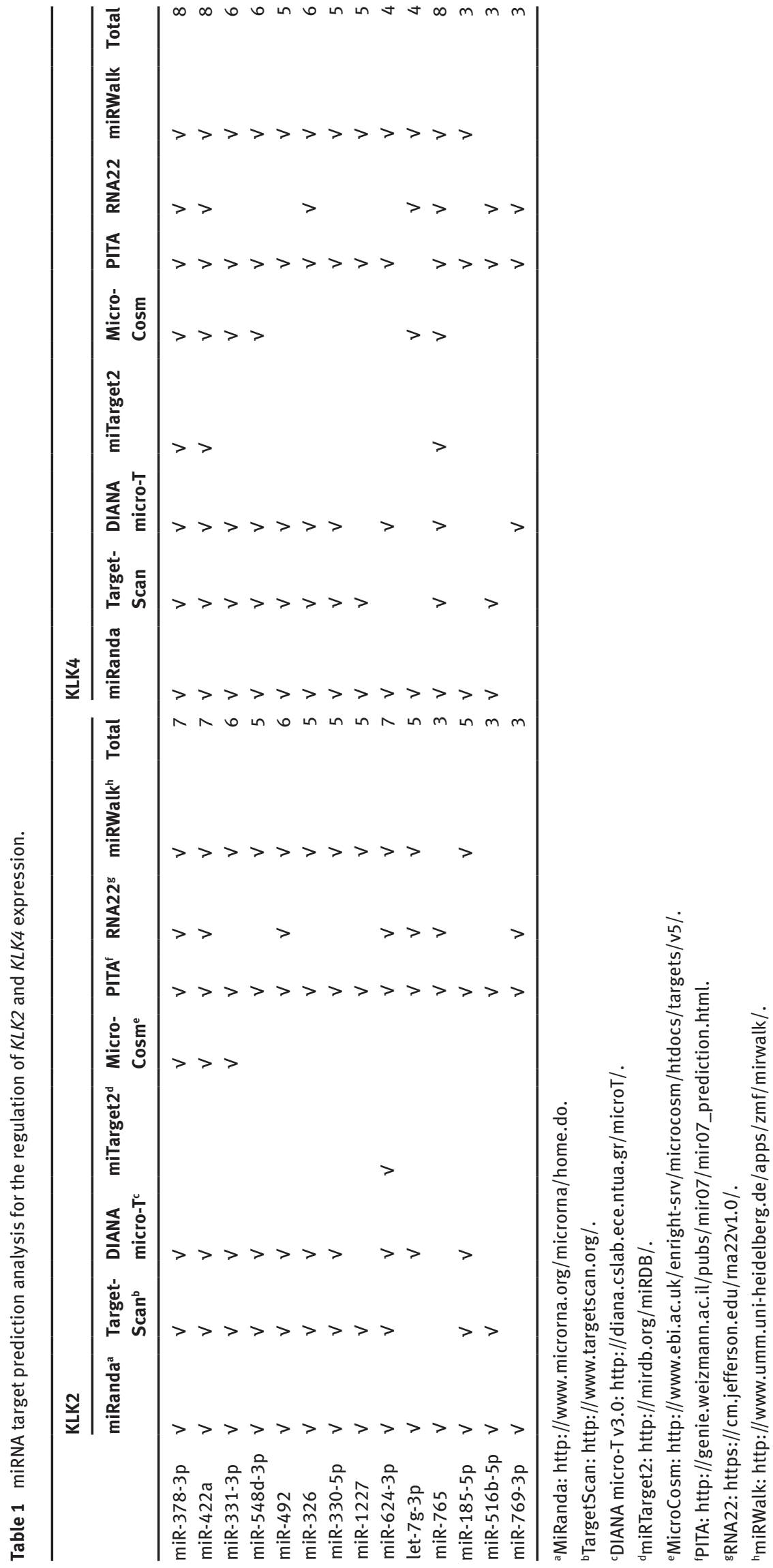



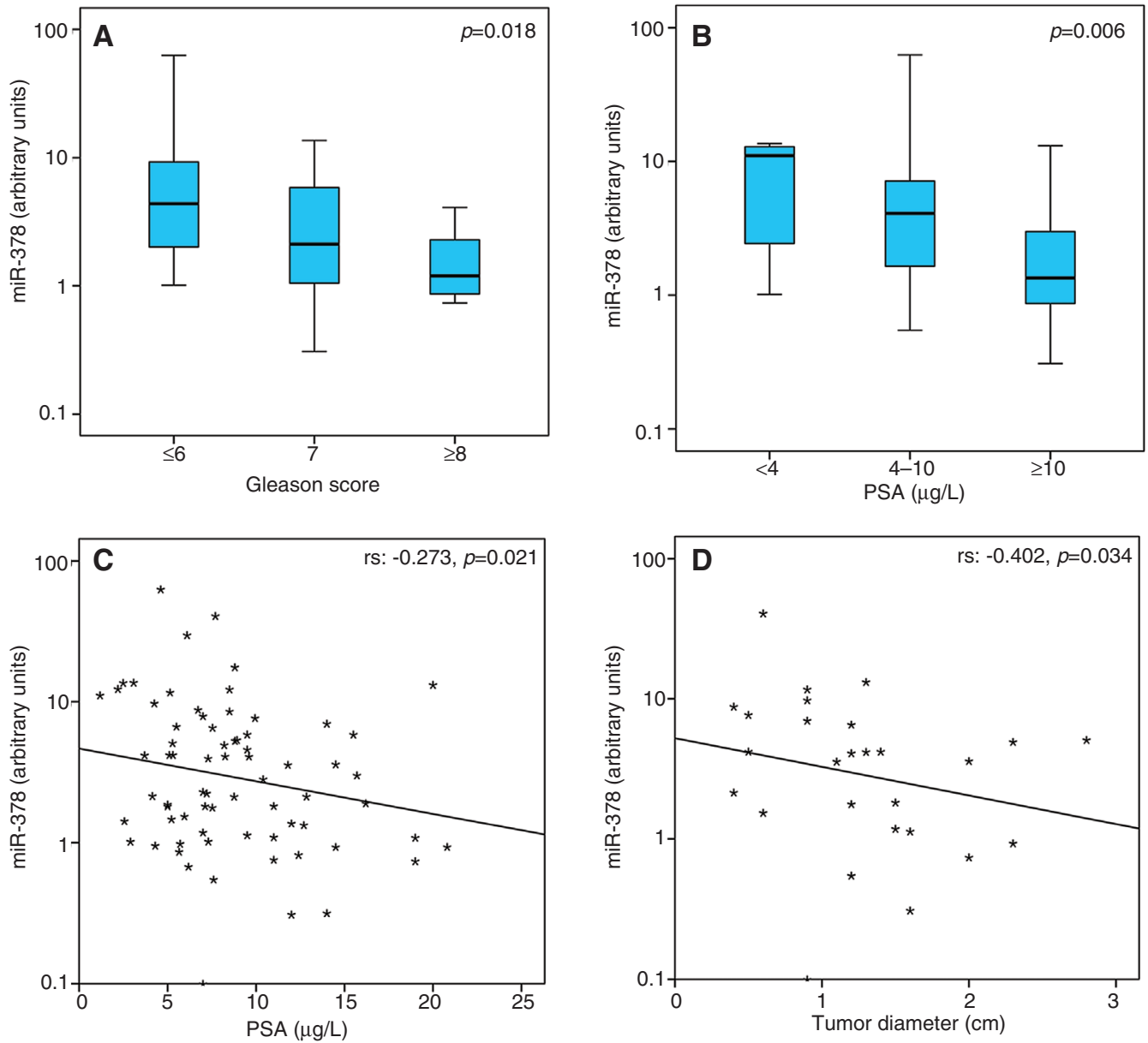

Figure $1 \mathrm{miR}-378$ expression levels are reduced in prostate cancer patients of higher Gleason score, elevated serum PSA levels, and larger tumors.

Expression analysis of miR-378 levels with Gleason score (A), serum PSA concentration (B and C), and larger tumor diameter (D). $p$-Value calculated by 'Kruskal-Wallis test' (A and B) and 'Spearman correlation' ( $C$ and D). $r_{s}$, Spearman correlation coefficient.

\section{Downregulation of miR-378 highlights the poor disease-free survival of the patients and improves risk stratification}

The prognostic significance of miR-378 for patients' outcome following radical prostatectomy was assessed by Kaplan-Meier survival curves (Figure 2A) and Cox regression analysis (Table 2). Although not statistically significant, Kaplan-Meier curves $(p=0.070)$ and univariate Cox analysis (HR: 1.911; 95\% CI: 0.932-3.921; $p=0.077$ ) showed the unfavorable disease-free survival (DFS) and the higher risk for biochemical relapse of the miR-378(-) PCa patients compared to the miR-378(+) ones.

In order to improve patients' risk stratification, the prognostic significance of miR-378 was further analyzed in combination with patients' Gleason score and tumor stage. As indicated by Kaplan-Meier DFS curves, a more accurate prediction of the DFS expectancy following radical prostatectomy was achieved for the patients of the same Gleason score or tumor stage cohorts. More precisely, grouping patients according to Gleason score, miR-378 loss was able to distinguish the patients with worse DFS outcome for both $\leq 3+4$ and $\geq 4+3$ groups (Figure $2 \mathrm{~B}$ ). Similarly, miR-378 loss was demonstrated to predict the poor DFS for both $\leq$ pT2c and $\geq$ pT3a groups compared to patients with miR-378 overexpression of the same cohorts (Figure 2C).

These findings clearly underscore the unfavorable outcome of the patients with reduced miR-378 levels at the time of treatment and the beneficial impact of miR-378 on the improvement of patients' risk stratification. Additionally, the ability of miR-378 loss to distinguish the patients with worse DFS outcome in the same Gleason score or tumor stage cohorts prompted us to study miR-378 prognostic significance in each individual recurrence risk groups. 

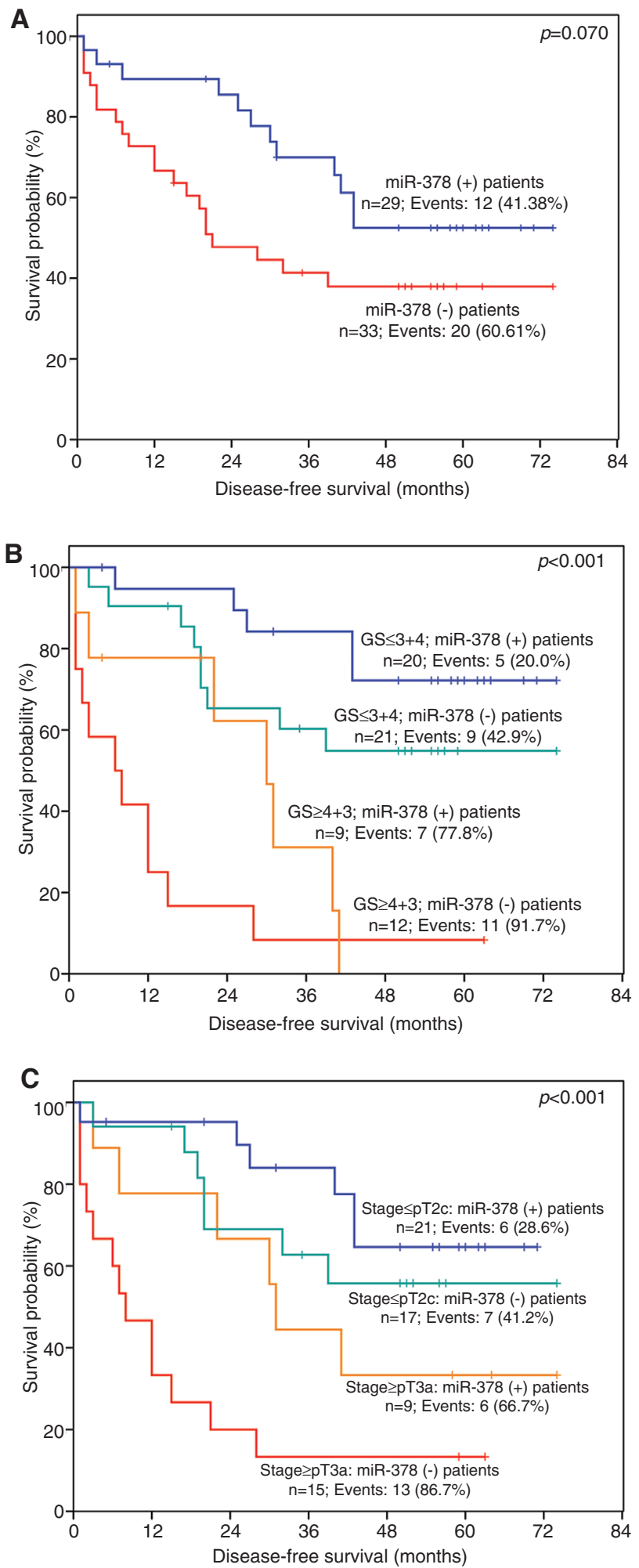

Figure 2 miR-378 downregulation in prostate cancer increases the risk for disease relapse and improves patients' risk stratification according to Gleason score and tumor stage.

Kaplan-Meier curves for the disease-free survival (DFS) of the prostate cancer patients according to miR-378 levels (A), Gleason score and miR-378 levels (B), and tumor stage and miR-378 levels (C). $p$-Value calculated by 'Log Rank test'. GS, Gleason score.

\section{Loss of miR-378 predicts the short-term relapse of the high-recurrence risk patients}

NCCN guidelines for PCa were used for the classification of the patients according to their recurrence risk (Mohler et al., 2010). Kaplan-Meier analysis, as well as Cox regression analysis (Table 3), was conducted in order to study miR-378a prognostic value for each cohort.

Regarding 'high'- and 'very high'-recurrence risk patients $(\geq \mathrm{pT3}$ a or Gleason score $8-10$ or PSA $>20 \mu \mathrm{g} / \mathrm{l}$; Supplemental Table 1), a significantly $(p=0.030)$ higher risk for short-term relapse was observed in the patients with reduced miR-378 levels (Figure 3A). In particular, a significantly shorter DFS expectancy was demonstrated for the miR-378(-) high- and very high-risk patients compared to the miR-378(+) ones. For the same group of patients, risk-group stratification was not efficient enough to predict the DFS of the treated patients ( $p=0.275$; Figure $3 \mathrm{~B}$ ), strengthening, in this way, the prognostic potential of miR-378. Studying miR-378 levels in low- and intermediate-recurrence risk patients (Supplemental Table 1), similar DFS intervals were demonstrated for miR378(-) and miR-378(+) patients.

The significance of the miR-378 expression in the prognosis of 'high'- and 'very high'-recurrence risk patients was also supported by Cox regression survival analysis. A significantly higher risk to relapse (HR: 2.824; $95 \%$ CI: $1.051-7.585 ; p=0.040$ ) was observed in the miR-378(-) patients compared to miR-378(+) patients by univariate models. For the same patients' group, only Gleason score was proved to have a statistically significant prognostic value for the prediction of biochemical relapse. Finally, multivariate Cox regression models revealed prognostic value of miR-378 levels for the prediction of patients' DFS following treatment (HR: 4.792; 95\% CI: 1.130-17.522; $p=0.018$ ) independent from Gleason score, tumor stage, PSA, DRE, and age. Summarizing, miR-378 loss in PCa represents an independent prognostic marker for the prediction of high- and very high-recurrence risk patients' short-term relapse.

\section{Discussion}

Since their discovery, miRNAs have emerged as powerful posttranscriptional gene expression regulatory mediators (Bartel, 2004), and deregulated miRNA levels have been documented in the majority of human malignancies (Calin and Croce, 2006; Bartels and Tsongalis, 2009). Therefore, the study of miRNAs' role in several 
Table 2 Cox proportional regression analysis for the prediction of the PCa patients' disease-free survival.

\begin{tabular}{|c|c|c|c|c|c|c|}
\hline \multicolumn{7}{|l|}{ PCa patients } \\
\hline \multirow[t]{2}{*}{ Covariant } & \multicolumn{3}{|c|}{ Univariate analysis } & \multicolumn{3}{|c|}{ Multivariate analysi } \\
\hline & HR & $95 \% \mathrm{Cl}^{\mathrm{a}}$ & $p$-Value ${ }^{b}$ & HR & $95 \% \mathrm{Cl}^{\mathrm{a}}$ & $p$-Value ${ }^{b}$ \\
\hline \multicolumn{7}{|l|}{ miR-378 } \\
\hline Positive & 1.00 & & & 1.00 & & \\
\hline Negative & 1.911 & $0.932-3.921$ & 0.077 & 1.722 & $0.818-3.624$ & 0.152 \\
\hline Gleason score & 2.027 & $1.480-2.777$ & $<0.001$ & 1.903 & $1.250-2.897$ & 0.003 \\
\hline Clinical stage & 1.506 & $1.178-1.924$ & 0.001 & 1.000 & $0.695-1.437$ & 0.998 \\
\hline PSA & 1.092 & $1.035-1.151$ & 0.001 & 1.035 & $0.968-1.106$ & 0.315 \\
\hline Age & 0.996 & $0.944-1.051$ & 0.879 & 0.990 & $0.935-1.049$ & 0.738 \\
\hline DRE & 2.276 & $1.100-4.710$ & 0.027 & 1.671 & $0.758-3.684$ & 0.203 \\
\hline
\end{tabular}

${ }^{\mathrm{a}}$ Confidence interval of the estimated $H R$.

${ }^{\mathrm{b}}$ Test for trend.

cancer-related molecular cascades would aid to the elucidation of the clinical significance for PCa patients' management (Schaefer et al., 2010b; Fendler et al., 2011; Larne et al., 2013; Lichner et al., 2013).

Using miRNA-target prediction programs, miR-378-3p was found to be the most frequently predicted miRNA candidate able to regulate both KLK2 and KLK4 expression. This is in agreement with miRNA microarray-based results showing downregulated miR-378 levels in PCa (Martens-Uzunova et al., 2012), opposite to the elevated expression of its KLK2 and KLK4 targets (Avgeris et al., 2012). Among the different variants of miR-378-3p (miR378a-j and miR-422a), miR-378a-3p (previously annotated as miR-378 or miR-422b) is the predominantly expressed variant of the miR-378/422a family, encoded by MIR378A, an intronic miRNA encoded gene embedded within the first intron of PPARGC1B gene in the $5 \mathrm{q} 32$ chromosomal locus.
Although MIR378A has been studied in various human malignancies, its function and expression profile has not been fully elucidated yet. MIR378A overexpression in U87 glioblastoma and MT-1 breast carcinoma cells was essential for cell survival, tumor growth, and angiogenesis, through the downregulation of SuFu and Fus-1 tumor suppressors by miR-378a-5p (Lee et al., 2007), while miR-378a-3p was found to be crucial for Myc-mediated cell transformation, following the silencing of the TOB2 antiproliferative factor (Feng et al., 2011). Regarding non-small cell lung cancer, mir-378a overexpression in NCI-H292 cells resulted to downregulation of HMOX1 and increased cell proliferation and migration (Skrzypek et al., 2013), while higher miR-378 levels were associated with patients' brain metastasis, tumor growth, and angiogenesis of A549 xenografts (Chen et al., 2012).

However, studies of miR-378 in the pathophysiology of the heart question its oncogenic properties. In particular,

Table 3 Cox proportional regression analysis for the prediction of the high- and very high-recurrence risk PCa patients' disease-free survival.

\begin{tabular}{|c|c|c|c|c|c|c|}
\hline \multicolumn{7}{|c|}{ High- and very high-recurrence risk PCa patients } \\
\hline \multirow[t]{2}{*}{ Covariant } & & \multicolumn{2}{|c|}{ Univariate analysis } & \multicolumn{3}{|c|}{ Multivariate analysis } \\
\hline & HR & $95 \% \mathrm{Cl}^{\mathrm{a}}$ & $p$-Value ${ }^{b}$ & HR & $95 \% \mathrm{Cl}^{\mathrm{a}}$ & $p$-Value ${ }^{b}$ \\
\hline \multicolumn{7}{|l|}{$\operatorname{miR}-378$} \\
\hline Positive & 1.00 & & & 1.00 & & \\
\hline Negative & 2.824 & $1.051-7.585$ & 0.040 & 4.792 & $1.310-17.522$ & 0.018 \\
\hline Gleason score & 1.737 & $1.084-2.783$ & 0.022 & 1.878 & $1.104-3.196$ & 0.020 \\
\hline Clinical stage & 1.718 & $0.751-3.931$ & 0.200 & 0.795 & $0.341-1.854$ & 0.596 \\
\hline PSA & 1.046 & $0.972-1.125$ & 0.230 & 0.976 & $0.888-1.072$ & 0.607 \\
\hline Age & 0.939 & $0.868-1.016$ & 0.119 & 0.936 & $0.864-1.013$ & 0.102 \\
\hline DRE & 2.779 & $0.802-9.637$ & 0.107 & 5.659 & $1.225-26.141$ & 0.026 \\
\hline
\end{tabular}

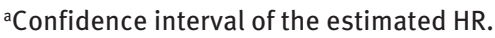

${ }^{\mathrm{b}}$ Test for trend. 
High \& Very high-recurrence risk PCa patients
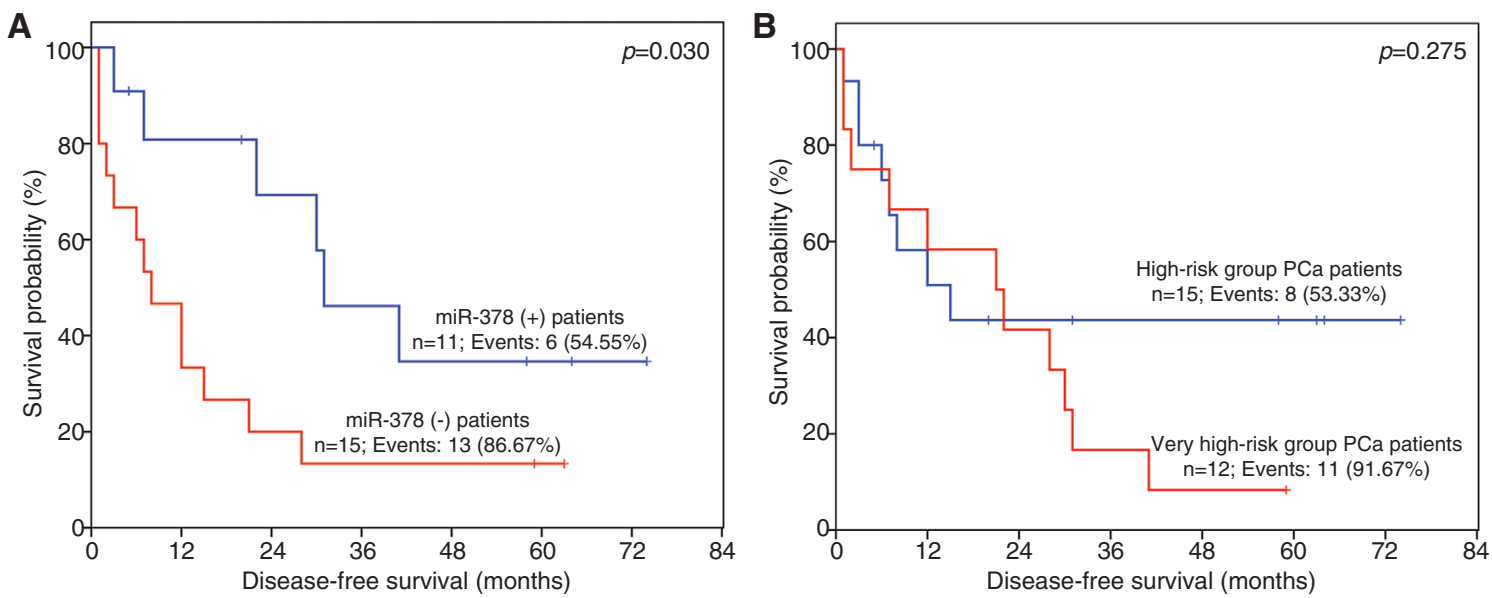

Figure 3 miR-378 loss in 'high'- and 'very high'-recurrence risk patients ( $\geq$ pT3a or Gleason score 8-10 or PSA $>20 \mu \mathrm{g} / \mathrm{l})$ predicts the shortterm disease relapse following radical prostatectomy.

Kaplan-Meier curves for the disease-free survival (DFS) of the 'high'- and 'very high'-risk patients according to miR-378 levels (A) and to NCCN guidelines risk-group stratification (B). $p$-Value calculated by 'Log Rank test’.

reduced miR-378 levels have been found in hypertrophic heart growth and cardiac failure (Ganesan et al., 2013; Nagalingam et al., 2013). These antihypertrophic activities of miR-378 are mainly exerted through the suppression of the MAPK/ERK and PI3K/AKT signaling pathways. More precisely, miR-378 overexpression blocks cardiac hypertrophy by preventing Ras activation and thus MAPK/ERK and PI3K/AKT downstream cascades, by targeting the GRB2 upstream mediator (Nagalingam et al., 2013), as well as MAPK1 (ERK2), IGF1R, and KSR1 members of the MAPK pathway (Ganesan et al., 2013). The miR-378a-mediated regulation of IGF1R signaling is even more critical in PCa pathogenesis. Overexpression of miR-378 resulted to the translational inhibition of IGF1R and to apoptotic cell death, while miR-378 knockdown launches IGF1R-related Akt cascade (Knezevic et al., 2012).

In order to evaluate miR-378 clinical significance for PCa, we measured miR-378 levels in cancerous and benign prostate tissues. Decreased miR-378 levels were found in PCa patients compared to BPH control ones. In PCa patients, miR-378 loss was further correlated with high Gleason score, tumors with larger diameter, and elevated serum PSA levels. Motivated by the correlation of miR-378 loss with unfavorable prognostic markers, we analyzed the significance of miR-378 in the survival outcome of the patients. As expected, Cox regression analysis revealed a significantly higher risk for relapse of the patients with reduced miR-378 levels, and Kaplan-Meier DFS curves indicated the correlation of miR-378 loss with the shorter DFS intervals following radical prostatectomy. Interestingly, miR-378 quantification was shown to be able to improve significantly the Gleason score- or tumor stagebased risk stratification of the patients.

The prognostic significance of miR-378 was further evaluated in 'low'- and 'intermediate'-recurrence risk as well as in 'high'- and 'very high'-recurrence risk patients. Interestingly, the loss of miR-378 in high- and very highrisk groups of patients ( $\geq$ pT3a or Gleason score: 8-10 or PSA $>20 \mu \mathrm{g} / \mathrm{l}$ ) predicts their short-term relapse, highlighting the utility of miR-378 for a more accurate prognosis of high- and very high-risk group. Kaplan-Meier curves suggested significantly worse DFS of the patients displaying miR-378 loss, while univariate Cox regression analysis pointed out an approximately threefold higher risk for relapse. Multivariate models highlighted that the prediction of the short-term relapse of the PCa patients with miR378a loss and their poor DFS is independent of Gleason score, tumor stage, serum PSA levels, age, and DRE. These results not only support a possible central role of miR-378 in disease progression but also underscore miR-378 as a novel independent prognostic marker for 'high'- and 'very high'-recurrence risk cohort.

Although prostate carcinogenesis is not the result of a single molecular event, ligand-independent pathways (outlaw pathways) of AR overactivation, especially in the late disease stages, are essential for disease progression and aggressiveness. Regarding 'outlaw' activation of AR, receptor-tyrosine-kinases (RTK) signaling, mediated mainly by IGF1R, and IL6- and IL8-related signaling, triggers AR overactivation through PI3K/AKT, MAPK, and JAK/STAT3 pathways, while KLK2 and KLK4 have been found to enhance AR activity in an androgen-independent 
manner (Mavridis et al., 2014). Consequently, the ability of miR-378 to target KLK2 and KLK4, along with IGF1R signaling and key components of MAPK and AKT pathways, highlights a possible regulatory role of miR-378 upon AR signaling in prostate cells. This is in agreement with the observed, in our analysis, correlation of miR-378 downregulation with higher Gleason score and larger-diameter prostate tumors, as well as with the poor survival expectancy of the patients.

In conclusion, in the present study, we have identified in silico miR-378 as a common regulator of KLK2 and KLK4 PCa-related members of the KLK family and analyzed miR-378 clinical significance for the prognosis of PCa patients. Reduction of miR-378 levels was observed in PCa tissues compared to benign gland lesions. The downregulation of miR-378 expression was correlated with disease progression, as patients with higher Gleason score tumors and elevated serum PSA concentrations were found to have significantly lower miR-378 levels. This was further verified by the higher risk for relapse and the worse DFS of the patients with lower miR-378 expression. Finally, in high- and very high-recurrence risk patients, miR-378 loss was shown to predict the short-term relapse following treatment of PCa patients independent from Gleason score, tumor stage, PSA levels, and age. Overall, these findings highlight the reduced expression of miR-378 in $\mathrm{PCa}$, the correlation of miR-378 downregulation with high Gleason score tumors and elevated serum PSA levels, and finally the ability of miR-378 to predict the survival outcome of the treated patients, supporting, in this way, miR-378 potential use to improve PCa prognosis.

\section{Materials and methods}

\section{miRNA targeting prediction analysis}

MiRanda, TargetScan, DIANA micro-T v3.0, miRTarget2, MicroCosm, PITA, RNA22, and miRWalk online available programs (see Table 1 for links to the above-mentioned algorithms) were used for the prediction of KLK2- and KLK4-targeting miRNAs via miRecords (http:// www.mirecords.umn.edu/miRecords/), miRNA body map (http:// www.mirnabodymap.org/), and miRWalk (http://www.umm.uniheidelberg.de/apps/zmf/mirwalk/) databases. The analyses were performed using the databases' default parameters.

\section{Study population}

Prostate tissue specimens were obtained from 73 PCa patients who underwent radical retropubic prostatectomy (Supplemental Tables 2 and 3) and 64 transurethral or open prostatectomy treated benign prostate hyperplasia (BPH) patients. Focusing on PCa patients, a tissue sample of approximately $200 \mathrm{mg}$ was sectioned from the peripheral zone of prostate gland based on the preoperative features of the biopsy and on macroscopic findings. The sample was thereafter divided into two mirror-image specimens, one of which was tested by a pathologist, while the other one was immediately frozen in liquid nitrogen and stored at $-80^{\circ} \mathrm{C}$ until analysis. None of the patients included received hormonal therapy or radiotherapy prior to surgery. The last measurement of serum PSA prior to prostate biopsy and diagnosis, approximately $1-2$ months prior to radical prostatectomy, was used in our analysis. Our study was approved by the ethics committee of 'Laiko General Hospital' and performed with respect to the ethical standards of the Declaration of Helsinki, as revised in 2008.

The follow-up data from 62 PCa patients were used for the assessment of miR-378 prognostic significance for prostate cancer, while 11 PCa patients were excluded from the monitoring plan due to either unclear follow-up data or the administration of adjuvant therapy before biochemical recurrence. PCa patients' were followedup according to European Association of Urology (EAU) guidelines, and biochemical relapse was defined by two consecutive measurements of serum PSA $\geq 0.2 \mu \mathrm{g} / \mathrm{l}$. The National Comprehensive Cancer Network (NCCN) guidelines for PCa (Supplemental Table 1) were followed for the evaluation of patients' recurrence risk (Mohler et al., 2010).

\section{Extraction and polyadenylation of total RNA}

Total RNA was isolated using TRI-Reagent (Molecular Research Center, Inc., Cincinnati, OH, USA) according to manufacturer's instructions, following the pulverization of $60-120 \mathrm{mg}$ of tissue specimen. Total RNA concentration and purity were determined spectrophotometrically at 260 and $280 \mathrm{~nm}$, while RNA integrity was evaluated by agarose gel electrophoresis. Thereafter, $1 \mu \mathrm{g}$ of total RNA was polyadenylated at the $3^{\prime}$-end in a $15-\mu \mathrm{l}$ reaction at $37^{\circ} \mathrm{C}$ for 60 min using $800 \mu \mathrm{M}$ ATP and $1 \mathrm{U}$ of Escherichia coli Poly(A) Polymerase (New England Biolabs Inc., Ipswich, MA, USA). Enzyme heat inactivation was performed at $65^{\circ} \mathrm{C}$ for $15 \mathrm{~min}$.

\section{First-strand cDNA synthesis}

The polyadenylated total RNA was reversed transcribed in a $20-\mu \mathrm{l}$ reaction containing $50 \mathrm{U}$ MMLV reverse transcriptase (Invitrogen, Carlsbad, CA, USA), 40 U recombinant ribonuclease inhibitor (Invitrogen), and $0.25 \mu \mathrm{m}$ poly(T) adapter $5^{\prime}$-GCGAGCACAGAATTAATACGACTCACTATAGGTTTTTTTTTTTTVN-3' ( $=\mathrm{G}, \mathrm{A}, \mathrm{C}$ and $\mathrm{N}=\mathrm{G}, \mathrm{A}, \mathrm{T}$, C) at $37^{\circ} \mathrm{C}$ for $60 \mathrm{~min}$ (Avgeris et al., 2013; Mavridis et al., 2013). The reverse transcriptase was inactivated by heating at $70^{\circ} \mathrm{C}$ for $15 \mathrm{~min}$.

\section{Quantitative real-time PCR (qPCR)}

Specific forward primers for the miR-378a and the small nucleolar RNA, C/D box 48 (SNORD48), also known as RNU48, were designed based on their published sequences (NCBI RefSeq accession number: NR_029870.1 and NR_002745.1, respectively) and in silico specificity evaluation. Moreover, a universal reverse primer, able to hybridize to 
the above-mentioned poly(T) adapter, was also synthesized. The combination of the $5^{\prime}$-GGACTGGACTTGGAGTCAGAA-3' forward primer for miR-378a or the 5'-TGATGATGACCCCAGGTAACTCT-3' forward primer for SNORD48 with the universal reverse primer $5^{\prime}$-GCGAGCACAGAATTAATACGAC-3', amplified a 67-bp miR-378- and a 105-bp SNORD48specific amplicon, respectively (Avgeris et al., 2013; Mavridis et al., 2013).

The qPCR assay was performed in the 7500 Real-Time PCR System (Applied Biosystems, Carlsbad, CA, USA), and the 10- $\mu$ l reaction consists of Kapa SYBR ${ }^{\circledR}$ Fast Universal 2x qPCR Master Mix (Kapa Biosystems, Inc., Woburn, MA, USA), 200 nM of each PCR primer, and 0.2 ng of cDNA. The thermal protocol comprised of a 3-min polymerase activation step at $95^{\circ} \mathrm{C}, 40$ cycles of denaturation at $95^{\circ} \mathrm{C}$ for $15 \mathrm{~s}$, and a primer annealing and extension step at $60^{\circ} \mathrm{C}$ for $1 \mathrm{~min}$. Following the amplification, melting curve analysis and agarose gel electrophoresis were used for the determination of specific reaction products compared to nonspecific ones or primer dimmers. Using serial dilutions of a control cDNA as template, calibration curves for miR-378 and SNORD48 amplification were generated covering six orders of magnitude (Supplemental Figure 3). The linear increases of miR-378 $\left(y=-3.47 x+43.49 ; r^{2}=0.991\right)$ and SNORD48 $\left(y=-3.32 x+38.02 ; r^{2}=0.999\right)$ amplification highlighted the $94.3 \%$ and $99.9 \%$ reaction efficiencies, respectively, as well as the absence of PCR inhibition by the template.

Relative quantification (RQ) of miR-378 was performed using the $2^{-\Delta \Delta C_{r}}$ method, where the LNCaP prostate cancer cell line was used as our assay calibrator and SNORD48 as an endogenous reference control (Larne et al., 2009). Duplicate reactions were performed for each tested sample, and the average $C_{t}$ was calculated for the quantification analysis. No template controls, reverse transcriptase-negative controls, Poly(A) polymerase-negative controls, and DNA template controls generated undetectable $C_{t}$ or $>36$.

\section{Statistical analysis}

The analysis of miR-378 levels between PCa and BPH patients was performed using the Mann-Whitney $U$-test. Univariate logistic regression analysis, calculating $\log _{10}$ miR-378 odds ratio (OR) and ROC curve analysis were used for the evaluation of miR-378 ability to discriminate PCa from $\mathrm{BPH}$ patients.

Differences in miR-378 expression levels among patients of different Gleason score, tumor stages, PSA group, or digital rectal examination (DRE) were analyzed using Mann-Whitney $U$ and Kruskal-Wallis tests. The correlation of miR-378 expression with PCa patients' PSA levels and tumor diameter was assessed by Spearman's correlation coefficient $\left(\mathrm{r}_{\mathrm{s}}\right)$. Using the X-tile algorithm, the 3.12 RQ units of miR-378 (equal to the 52nd percentile of the PCa patients' cohort) were adopted as an optimal cutoff to classify PCa patients into miR-378(+) and miR-378(-) cohorts, expressing higher and lower miR-378 levels, respectively.

The prognostic significance of miR-378 was evaluated by KaplanMeier survival analysis, using log rank test, and Cox proportional hazards regression models. Univariate and multivariate Cox regression models were employed for the calculation of the hazard ratio (HR) of miR-378, Gleason score, tumor stage, serum PSA, patient's age, and DRE.

Acknowledgments: This research has been co-financed by the European Union (European Social Fund-ESF) and Greek national funds through the Operational Program 'Education and Lifelong Learning' of the National Strategic Reference Framework (NSRF)-Research Funding Program: THALES, Investing in knowledge society through the European Social Fund (UoA - BIOPROMO, MIS 377046).

\section{References}

Andriole, G.L., Crawford, E.D., Grubb, R.L., 3rd, Buys, S.S., Chia, D., Church, T.R., Fouad, M.N., Gelmann, E.P., Kvale, P.A., Reding, D.J., et al. (2009). Mortality results from a randomized prostate-cancer screening trial. N. Engl. J. Med. 360, 1310-1319.

Avgeris, M., Mavridis, K., and Scorilas, A. (2010). Kallikrein-related peptidase genes as promising biomarkers for prognosis and monitoring of human malignancies. Biol. Chem. 391, 505-511.

Avgeris, M., Stravodimos, K., and Scorilas, A. (2011). Kallikreinrelated peptidase 4 gene (KLK4) in prostate tumors: quantitative expression analysis and evaluation of its clinical significance. Prostate 71, 1780-1789.

Avgeris, M., Mavridis, K., and Scorilas, A. (2012). Kallikreinrelated peptidases in prostate, breast, and ovarian cancers: from pathobiology to clinical relevance. Biol. Chem. 393, 301-317.

Avgeris, M., Stravodimos, K., Fragoulis, E.G., and Scorilas, A. (2013). The loss of the tumour-suppressor miR-145 results in the shorter disease-free survival of prostate cancer patients. $\mathrm{Br}$. J. Cancer 108, 2573-2581.

Bartel, D.P. (2004). MicroRNAs: genomics, biogenesis, mechanism, and function. Cell 116, 281-297.

Bartels, C.L. and Tsongalis, G.J. (2009). MicroRNAs: novel biomarkers for human cancer. Clin. Chem. 55, 623-631.

Borgono, C.A., Michael, I.P., and Diamandis, E.P. (2004). Human tissue kallikreins: physiologic roles and applications in cancer. Mol. Cancer Res. 2, 257-280.

Calin, G.A. and Croce, C.M. (2006). MicroRNA signatures in human cancers. Nat. Rev. Cancer 6, 857-866.

Chen, L.T., Xu, S.D., Xu, H., Zhang, J.F., Ning, J.F., and Wang, S.F. (2012). MicroRNA-378 is associated with non-small cell lung cancer brain metastasis by promoting cell migration, invasion and tumor angiogenesis. Med. Oncol. 29, 1673-1680.

Chow, T.F., Crow, M., Earle, T., El-Said, H., Diamandis, E.P., and Yousef, G.M. (2008). Kallikreins as microRNA targets: an in silico and experimental-based analysis. Biol. Chem. 389, 731-738.

Darson, M.F., Pacelli, A., Roche, P., Rittenhouse, H.G., Wolfert, R.L., Young, C.Y., Klee, G.G., Tindall, D.J., and Bostwick, D.G. (1997). Human glandular kallikrein 2 (hK2) expression in prostatic intraepithelial neoplasia and adenocarcinoma: a novel prostate cancer marker. Urology 49, 857-862.

Fendler, A., Stephan, C., Yousef, G.M., and Jung, K. (2011). MicroRNAs as regulators of signal transduction in urological tumors. Clin. Chem. 57, 954-968.

Feng, M., Li, Z., Aau, M., Wong, C.H., Yang, X., and Yu, Q. (2011). $\mathrm{Myc} / \mathrm{miR}-378 / \mathrm{TOB} 2 /$ cyclin D1 functional module regulates oncogenic transformation. Oncogene 30, 2242-2251. 
Freedland, S.J. (2011). Screening, risk assessment, and the approach to therapy in patients with prostate cancer. Cancer 117, 1123-1135.

Ganesan, J., Ramanujam, D., Sassi, Y., Ahles, A., Jentzsch, C., Werfel, S., Leierseder, S., Loyer, X., Giacca, M., Zentilin, L., et al. (2013). MiR-378 controls cardiac hypertrophy by combined repression of mitogen-activated protein kinase pathway factors. Circulation 127, 2097-2106.

Herrala, A.M., Porvari, K.S., Kyllonen, A.P., and Vihko, P.T. (2001). Comparison of human prostate specific glandular kallikrein 2 and prostate specific antigen gene expression in prostate with gene amplification and overexpression of prostate specific glandular kallikrein 2 in tumor tissue. Cancer 92, 2975-2984.

Klokk, T.I., Kilander, A., Xi, Z., Waehre, H., Risberg, B., Danielsen, H.E., and Saatcioglu, F. (2007). Kallikrein 4 is a proliferative factor that is overexpressed in prostate cancer. Cancer Res. 67, 5221-5230.

Knezevic, I., Patel, A., Sundaresan, N.R., Gupta, M.P., Solaro, R.J., Nagalingam, R.S., and Gupta, M. (2012). A novel cardiomyocyte-enriched microRNA, miR-378, targets insulin-like growth factor 1 receptor: implications in postnatal cardiac remodeling and cell survival. J. Biol. Chem. 287, 12913-12926.

Larne, O., Edsjo, A., Bjartell, A., and Ceder, Y. (2009). Development of a miRNA assay for prostate cancer detection. Eur. Urol. Suppl. 8, S316.

Larne, O., Martens-Uzunova, E., Hagman, Z., Edsjo, A., Lippolis, G., den Berg, M.S., Bjartell, A., Jenster, G., and Ceder, Y. (2013). miQ-a novel microRNA based diagnostic and prognostic tool for prostate cancer. Int. J. Cancer 132, 2867-2875.

Lawrence, M.G., Lai, J., and Clements, J.A. (2010). Kallikreins on steroids: structure, function, and hormonal regulation of prostate-specific antigen and the extended kallikrein locus. Endocr. Rev. 31, 407-446.

Lee, D.Y., Deng, Z., Wang, C.H., and Yang, B.B. (2007). MicroRNA-378 promotes cell survival, tumor growth, and angiogenesis by targeting SuFu and Fus-1 expression. Proc. Natl. Acad. Sci. USA 104, 20350-20355.

Lichner, Z., Fendler, A., Saleh, C., Nasser, A.N., Boles, D., Al-Haddad, S., Kupchak, P., Dharsee, M., Nuin, P.S., Evans, K.R., et al. (2013). MicroRNA signature helps distinguish early from late biochemical failure in prostate cancer. Clin. Chem. 59, 1595-1603.

Martens-Uzunova, E.S., Jalava, S.E., Dits, N.F., van Leenders, G.J., Moller, S., Trapman, J., Bangma, C.H., Litman, T., Visakorpi, T., and Jenster, G. (2012). Diagnostic and prognostic signatures from the small non-coding RNA transcriptome in prostate cancer. Oncogene 31, 978-991.
Mavridis, K. and Scorilas, A. (2010). Prognostic value and biological role of the kallikrein-related peptidases in human malignancies. Future Oncol. 6, 269-285.

Mavridis, K., Stravodimos, K., and Scorilas, A. (2013). Downregulation and prognostic performance of microRNA 224 expression in prostate cancer. Clin. Chem. 59, 261-269.

Mavridis, K., Avgeris, M., and Scorilas, A. (2014). Targeting kallikrein-related peptidases in prostate cancer. Exp. Opin. Ther. Targets 18, 365-383.

Mohler, J., Bahnson, R.R., Boston, B., Busby, J.E., D’Amico, A., Eastham, J.A., Enke, C.A., George, D., Horwitz, E.M., Huben, R.P., et al. (2010). NCCN clinical practice guidelines in oncology: prostate cancer. J. Natl. Compr. Canc. Netw. 8, 162-200.

Nagalingam, R.S., Sundaresan, N.R., Gupta, M.P., Geenen, D.L., Solaro, R.J., and Gupta, M. (2013). A cardiac-enriched microRNA, miR-378, blocks cardiac hypertrophy by targeting Ras signaling. J. Biol. Chem. 288, 11216-11232.

Pasic, M.D., Olkhov, E., Bapat, B., and Yousef, G.M. (2012). Epigenetic regulation of kallikrein-related peptidases: there is a whole new world out there. Biol. Chem. 393, 319-330.

Porkka, K.P., Pfeiffer, M.J., Waltering, K.K., Vessella, R.L., Tammela, T.L., and Visakorpi, T. (2007). MicroRNA expression profiling in prostate cancer. Cancer Res. 67, 6130-6135.

Schaefer, A., Jung, M., Mollenkopf, H.J., Wagner, I., Stephan, C., Jentzmik, F., Miller, K., Lein, M., Kristiansen, G., and Jung, K. (2010a). Diagnostic and prognostic implications of microRNA profiling in prostate carcinoma. Int. J. Cancer 126, 1166-1176.

Schaefer, A., Stephan, C., Busch, J., Yousef, G.M., and Jung, K. (2010b). Diagnostic, prognostic and therapeutic implications of microRNAs in urologic tumors. Nat. Rev. Urol. 7, 286-297.

Schroder, F.H., Hugosson, J., Roobol, M.J., Tammela, T.L., Ciatto, S., Nelen, V., Kwiatkowski, M., Lujan, M., Lilja, H., Zappa, M., et al. (2009). Screening and prostate-cancer mortality in a randomized European study. N. Engl. J. Med. 360, 1320-1328.

Skrzypek, K., Tertil, M., Golda, S., Ciesla, M., Weglarczyk, K., Collet, G., Guichard, A., Kozakowska, M., Boczkowski, J., Was, H., et al. (2013). Interplay between heme oxygenase-1 and miR-378 affects non-small cell lung carcinoma growth, vascularization, and metastasis. Antioxid. Redox Signal. 19, 644-660.

Wach, S., Nolte, E., Szczyrba, J., Stohr, R., Hartmann, A., Orntoft, T., Dyrskjot, L., Eltze, E., Wieland, W., Keck, B., et al. (2012). MicroRNA profiles of prostate carcinoma detected by multiplatform microRNA screening. Int. J. Cancer 130, 611-621.

Supplemental Material: The online version of this article (DOI: 10.1515/hsz-2014-0150) offers supplementary material, available to authorized users. 